\title{
Design and Implementation of a Facility for Discovering New Scintillator Materials
}

\author{
Stephen E. Derenzo, Fellow, IEEE, Martin S. Boswell, Edith Bourret-Courchesne, Rostyslav \\ Boutchko, Thomas F. Budinger, Member, IEEE, Andrew Canning, Stephen M. Hanrahan, Martin \\ Janecek, Member, IEEE, Qiyu Peng, Yetta Porter-Chapman, James Powell, Member, IEEE, \\ Christopher A. Ramsey, Scott E. Taylor, Lin-Wang Wang, Marvin J. Weber, and David S. Wilson
}

\begin{abstract}
We describe the design and operation of a highthroughput facility for synthesizing thousands of inorganic crystalline samples per year and evaluating them as potential scintillation detector materials. This facility includes a robotic dispenser, arrays of automated furnaces, a dual-beam X-ray generator for diffractometery and luminescence spectroscopy, a pulsed X-ray generator for time response measurements, computer-controlled sample changers, an optical spectrometer, and a network-accessible database management system that captures all synthesis and measurement data.
\end{abstract}

Index Terms-Scintillation detector material discovery, radioluminescence, phosphors, crystalline compounds.

\section{INTRODUCTION}

$\mathrm{T}_{2}^{\mathrm{H}}$ HERE are thousands of known crystalline compounds [1, 2] whose performances as scintillator radiation detectors (doped or undoped) have never been reported. It is likely that among these are scintillator materials with exceptional performance in terms of stopping power, luminosity, proportional response, speed, and cost. To discover such materials, we have designed and constructed a highthroughput facility for synthesizing inorganic compounds in crystalline powder form and for characterizing them as

Manuscript received June 29, 2007. This work was supported by the U.S. Department of Homeland Security and carried out at the Lawrence Berkeley National Laboratory under U.S. Department of Energy Contract No. DEAC02-05CH11231. This research used resources of the National Energy Research Scientific Computing Center, which is supported by the Office of Science of the U.S. Department of Energy under Contract No. DE-AC0205CH11231.

All authors are with the Lawrence Berkeley National Laboratory, Berkeley, CA 94720.

Stephen E. Derenzo, corresponding author, phone 510-486-4097; fax 510486-4768; e-mail SEDerenzo@lbl.gov

Edith Bourret-Courchesne, EDBourret@lbl.gov

Martin S. Boswell, MSBoswell@lbl.gov

Rostyslav Boutchko, RBuchko@1bl.gov

Thomas F. Budinger, TFBudinger@lbl.gov

Andrew Canning, ACanning@lbl.gov

Stephen M. Hanrahan, SMHanrahan@lbl.gov

Martin Janecek, MJanecek@lbl.gov

Qiyu Peng, QPeng@lbl.gov

Yetta Porter-Chapman, YDPorter-Chapman@lbl.gov

James D. Powell, JDPowell@lbl.gov

Christopher A. Ramsey, CARamsey@lbl.gov

Scott E. Taylor, SETaylor@lbl.gov

Lin-Wang Wang, LWWang@lbl.gov

Marvin J. Weber, MJWeber@bellsouth.net

David S.Wilson, DSWilson@1bl.gov potential scintillator detector materials. This facility consists of: 1) a robotic dispenser in a cabinet continuously purged with dry nitrogen gas for combining weights of starting compounds and dopants in desired ratios, 2) an array of controlled-atmosphere furnaces with differential thermal analysis to sense chemical reactions, 3) an X-ray diffractometer, 4) software for matching diffraction patterns and identifying crystalline phases, 5) an optical spectrometer to measure the emission spectrum during X-ray excitation, 6) an $80 \mathrm{ps}$ pulsed X-ray source and microchannel phototube for measuring scintillation light decay times, 7) an optical spectrometer for recording excitation and emission spectra, and 8) a network-accessible database management system (DBMS) that collects and organizes data generated by the other seven components and provides user-friendly interfaces for viewing, retrieving, and analyzing the data. Computercontrolled sample changers allow each of the three X-ray systems to measure over 50 samples per day. Materials exhibiting promising luminosity are candidates for synthesis and characterization as transparent solids. The discovery process using this facility is shown schematically in Fig. 1.

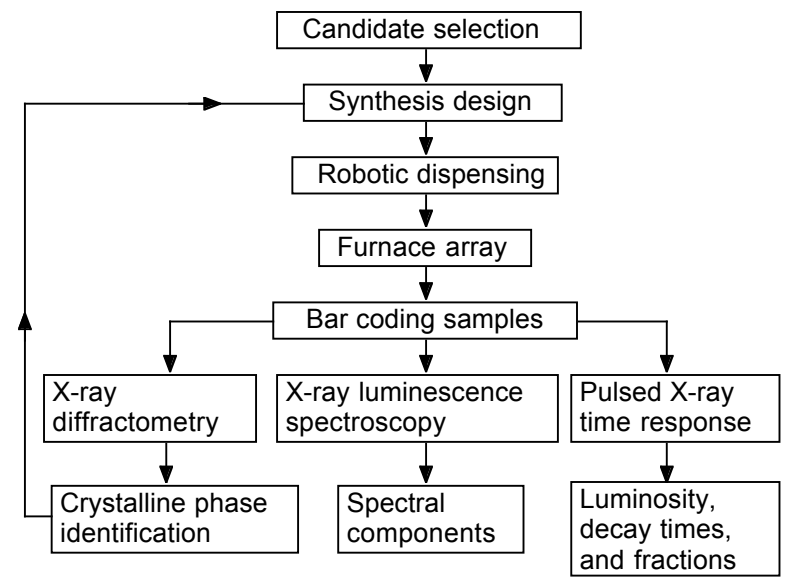

Fig. 1. Process for selecting candidates, synthesizing samples, and measuring their crystalline and X-ray-excited luminescence properties. 


\section{CAndidate Selection}

\section{A. Overall Objectives}

The objective of the facility is the synthesis of inorganic compounds in crystalline powder form and their characterization as potential scintillator detector materials.

Candidate compounds for the facility are selected on the basis of (1) good stopping power for gamma rays, which requires a high density and atomic number, and (2) avoidance of elements with long-lived natural radioisotopes such as $\mathrm{Rb}$, $\mathrm{Lu}$, and $\mathrm{K}$, which have 860,49 , and 31 disintegrations/s/g, respectively [3]. La is less radioactive (0.81 disintegrations $/ \mathrm{s} / \mathrm{g}$ ) and is not avoided.

Candidates for subsequent production as single crystals or transparent ceramics will be selected on the basis of high scintillation luminosity and short decay times. These samples can then be evaluated for 1) good proportional response and low optical absorption (which together with high luminosity result in good energy resolution), and 2) low cost in large sizes.

\section{B. Initial Selection}

For cerium and praseodymium activation our criteria for selecting candidate compounds include: 1) at least one metal cation and at least one anion from the following list $(\mathrm{O}, \mathrm{S}, \mathrm{Se}$, $\mathrm{Te}, \mathrm{F}, \mathrm{Cl}, \mathrm{Br}$, and I), 2) a divalent or trivalent site suitable for $\mathrm{Ce}^{3+}$ or $\mathrm{Pr}^{3+}$ substitution, 3) the absence of radioactive (e.g., $\mathrm{Rb}, \mathrm{K}$, and $\mathrm{Lu}$ ) or toxic (e.g., Be, $\mathrm{Tl}$, and $\mathrm{As}$ ) elements, 4) the absence of transition elements in optically active valence states (e.g., $\left.\left.\mathrm{Cr}^{3+}\right), 5\right)$ the absence of costly elements (e.g., $\mathrm{Ag}$, $\mathrm{Os}, \mathrm{Ir}, \mathrm{Pt}$, and $\mathrm{Au}$ ), 6) the absence of atomic transitions (e.g., $\mathrm{Bi}^{3+}$ ) or charge-transfer transitions (e.g., $\mathrm{WO}_{4}{ }^{2-}$ ) that interfere with $\mathrm{Ce}^{3+}$ activation, 7) at least one element with atomic number $>47$, and 8) a density above $4 \mathrm{gm} / \mathrm{cm}^{3}$. The requirements for $\mathrm{Eu}^{2+}$ activation are analogous.

Fig. 2 shows the cations and anions that are favored in our candidate selection process. Initial components are in the form of metal oxides, sulfides, and halides.

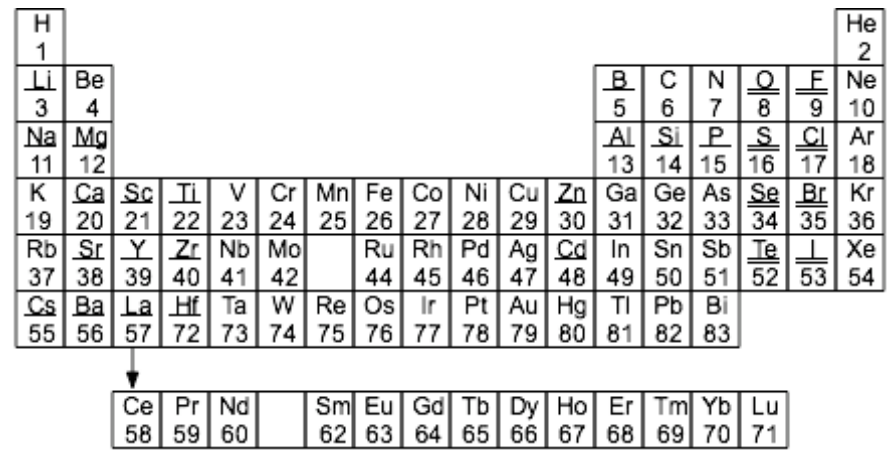

Fig. 2. Periodic table of the stable elements. Cations favored in candidate compounds for $\mathrm{Ce}^{3+}, \mathrm{Pr}^{3+}$, and $\mathrm{Eu}^{2+}$ doping are bordered by heavy lines. Anions similarly favored are bordered by double lines.

\section{First-Principles Calculations}

Scintillation in cerium-activated materials corresponds to the transition of an excited electron in the Ce $5 \mathrm{~d}$ state to the $\mathrm{Ce}$
$4 \mathrm{f}$ ground state. Using Density Functional Theory (DFT) to compute the ground state system $\mathrm{Ce}^{3+}$ and the excited state system $\left(\mathrm{Ce}^{3+}\right)^{*}$, we found that the following four factors are important in determining whether a material can be a bright Ce-activated scintillator. For a review of the DFT method, see [4].

\section{Factor 1: The size of the host material bandgap}

The number of electron-hole pairs produced by the incident gamma ray is inversely proportional to the band gap so that smaller band gaps can result in brighter scintillators. However, the gap has to be large enough to accommodate the Ce $5 \mathrm{~d}$ and 4f levels. This typically means the gap is $>4 \mathrm{eV}$ for oxides and halides, and somewhat less for sulfides.

Factor 2: The energy difference between the top of the valence band of the host crystal and the Ce 4 flevel

For efficient hole trapping on the ground state $\mathrm{Ce}^{3+}$, this difference should be small. The larger this energy difference, the less probable will be the hole capture at the $\mathrm{Ce}^{3+}$ site, as higher-energy phonons or multiple phonons will be required for the transition. If the $4 \mathrm{f}$ level is in the valence band, there will be no cerium scintillation because the $\mathrm{Ce}^{3+}$ ion cannot trap holes.

Factor 3: The energy difference between the excited electron in the system $\left(\mathrm{Ce}^{3+}\right) *$ and the bottom of the conduction band of the host crystal

If the excited electron is in the conduction band or is thermally excited into the conduction band, there will be no cerium scintillation because such electrons migrate and become trapped on defects or impurities.

Factor 4: The localization of the excited electron in the system $\left(\mathrm{Ce}^{3+}\right) *$ on the Ce ion

We have observed in yttrium-based systems (e.g., $\left.\mathrm{YAlO}_{3}: \mathrm{Ce}\right)$ that there is strong hybridization between the $\mathrm{Ce}$ $5 \mathrm{~d}$ and the $\mathrm{Y} 4 \mathrm{~d}$ states that form the conduction band of the host material. Therefore, to have a better measure of the probability of Ce scintillation, we compute the localization of the excited electron in the $\left(\mathrm{Ce}^{3+}\right)^{*}$ system rather than using Factor 3, which is not well defined for systems with hybridized bands. If this electron is not localized on the $\mathrm{Ce}^{4+}$ ion, there will be no Ce scintillation.

The computational procedure we have used for these calculations is as follows:

1) Get the atomic positions of the irreducible unit cell and the crystal symmetry group from the Inorganic Crystal Structure Database.

2) Relax the atomic positions and primitive cell size of the host material.

3) Generate a host crystal supercell, replace one of the atoms with a single $\mathrm{Ce}$ atom, and relax the atomic positions.

4) Perform a ground state Density Functional Theory, Local Density Approximation (DFT, LDA) band structure calculation to determine the position of the Ce $4 \mathrm{f}$ level with respect to the top of the valence band of the host crystal.

5) Perform a constrained DFT LDA calculation for the $\left(\mathrm{Ce}^{3+}\right) *$ state by forcing the occupancy of the $\mathrm{Ce} 4 \mathrm{f}$ states to be zero.

6) Relax the atomic positions of the $\left(\mathrm{Ce}^{3+}\right)^{*}$ system to 
determine the Stokes shift. This can lead to increased localization of the excited state on the $\mathrm{Ce}$ atom. From this calculation we determine the energy of the excited electron in the $\left(\mathrm{Ce}^{3+}\right)^{*}$ system relative to the bottom of the conduction band. In addition, we determine the localization of the excited state on the Ce ion by calculating the fraction of the excited electron's charge density that surrounds the Ce ion.

This approach has been validated by the study of known scintillators (e.g., $\mathrm{YAlO}_{3}: \mathrm{Ce}, \mathrm{LaF}_{3}: \mathrm{Ce}, \mathrm{LaCl}_{3}: \mathrm{Ce}, \mathrm{LaBr}_{3}: \mathrm{Ce}$, $\mathrm{LaI}_{3}: \mathrm{Ce}, \mathrm{CeBr}_{3}$, and $\mathrm{BaY}_{2} \mathrm{~F}_{8}: \mathrm{Ce}$ ) and non-scintillators (e.g., $\mathrm{Y}_{2} \mathrm{O}_{3}: \mathrm{Ce}, \mathrm{BiF}_{3}: \mathrm{Ce}$, and $\mathrm{LaAlO}_{3}: \mathrm{Ce}$ ) [5]. We are now confident that our approach has predictive value, and we used it to correctly predict that $\mathrm{Ba}_{2} \mathrm{YCl}_{7}$ : $\mathrm{Ce}$ would be a bright ceriumactivated scintillator before we synthesized and tested it.

Note that this approach is limited to crystalline materials whose crystal structure is known or whose structure can be determined reliably by ground-state relaxation. These materials are a subset of the compounds that we can explore experimentally. However, such calculations have additional value in predicting trends among families of related compounds and in providing insights to the factors that are important for successful scintillation.

\section{SAMPLE SYNTHESIS}

For each candidate compound, sample synthesis includes the following steps:

1) A literature search is performed to find previous reports of synthesis methods, photoluminescence, and X-ray or electron luminescence.

2) The reaction conditions are defined in terms of starting components, molar ratios, temperature vs. time profile, and atmosphere. Each mixture is assigned a sample number.

3) The robotic dispenser picks up bottles of starting components (e.g., metal oxides, sulfides, and halides) and deposits predetermined weights into crucibles. The parameters (molar ratios and quantities dispensed) are uploaded automatically into the on-line database and are associated with the sample number. Highest available purity materials are chosen (typically $99.999 \%$ metals basis). Samples of about 0.5 to 2 grams are usually produced with varying doping concentrations of cerium, praseodymium, or europium. The dispenser is in a cabinet that is continuously purged with dry nitrogen. The relative humidity is typically $2 \%$, which corresponds to an absolute humidity of $400 \mathrm{ppm}$ water vapor. The crucible receives a plastic cap for transfer to the furnace area. The sample is assigned a sample number in the database, and a bar code is attached to the plastic cap. Extremely hygroscopic materials, such as some halogens, are handled individually in a dry box.

4) The crucibles are loaded into the computer-controlled furnaces, and each cap is placed at the outside of the furnace. Currently the furnace array consists of $181200^{\circ} \mathrm{C}$ furnaces and $121600^{\circ} \mathrm{C}$ furnaces. The bar code of the furnace and bar code of the plastic cap are read and associated with the sample number in the on-line database. For air-sensitive samples, the transfer is made in an argon-filled container, and the furnace process tube is continuously purged with inert gas. Data from each furnace (atmosphere and temperature profile vs. time) are uploaded to the on-line database. Furnace control and temperature sensing are done using a custom LabVIEW program interfaced to the furnaces and thermocouples using Compact FieldPoint circuits (National Instruments, Inc., Austin, TX).

If the phase diagram of a compound is known, or if the solid-state reaction is documented in the literature, then the reaction temperature and time are chosen accordingly to produce that compound. If not, the reaction temperature is chosen below the melting point of the starting components, and the process time is 16 hours (overnight). It is then adjusted following the $\mathrm{x}$-ray diffraction analysis of the produced compounds until the desired phase is obtained. If only the starting components are present, the synthesis is repeated at a higher temperature and/or for a longer time. If decomposition has occurred (mass loss or dark product), the synthesis is repeated in a sealed ampoule. We have verified our synthesis protocols by synthesizing known scintillators.

To detect exothermic or endothermic reactions that occur during synthesis (e.g., re-crystallization, solid phase transitions, or solid-liquid transitions) a dual-well crucible holder (Fig. 3) is used to perform differential thermal analysis, where the temperature difference between the sample crucible and an empty crucible is recorded during processing.

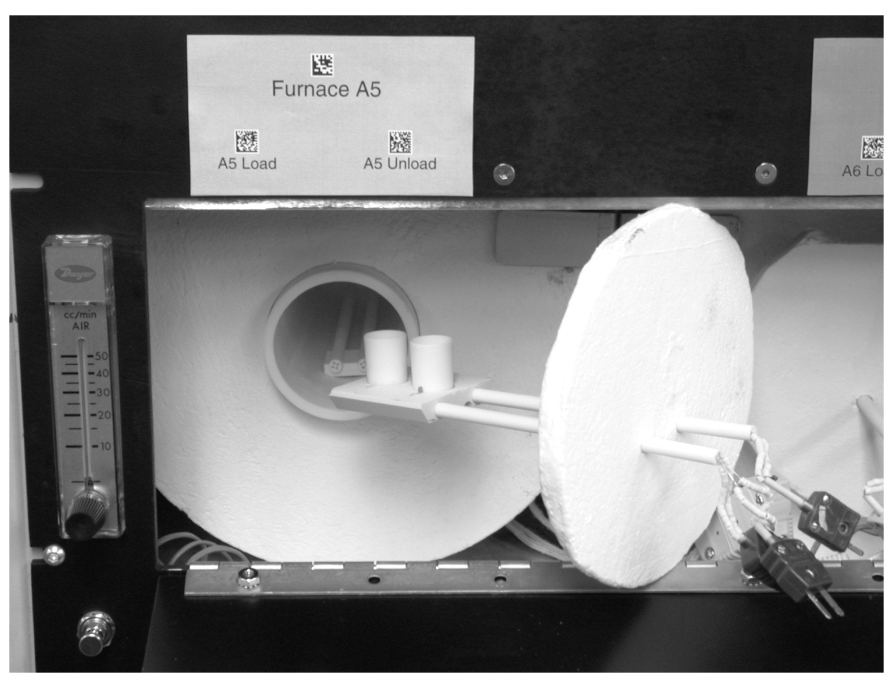

Fig. 3. Furnace and holder for two crucibles. Thermocouples in contact with the crucibles are wired to provide a differential thermal signal for detecting crystallization, solid phase transitions, and solid-liquid transitions.

\section{BARCODE TRACKING}

Processed samples are transferred from the furnace crucibles to sample holders that are identified with a $2 \mathrm{D}$ barcode adhesive label. These sample holders are shown in Fig. 4 and include nylon washers for X-ray diffraction measurements, and fused silica cuvettes for luminescence spectra and pulsed X-ray measurements. The barcodes code a unique 14-digit number using "DataMatrix" symbology, and each physical sample is given a different barcode. A handoperated barcode reader (shown in Fig. 4) is used to record the associations between the sample number and the different 
barcodes in the on-line database. Thereafter, the sample changers for the three measuring systems described below first read the 2D barcode, take data, and then automatically transfer the data to the on-line database. Human error is reduced because 1) the unique barcode labels affixed to the physical samples can be chosen in any order, and 2) samples can be loaded into the sample changers in any order.

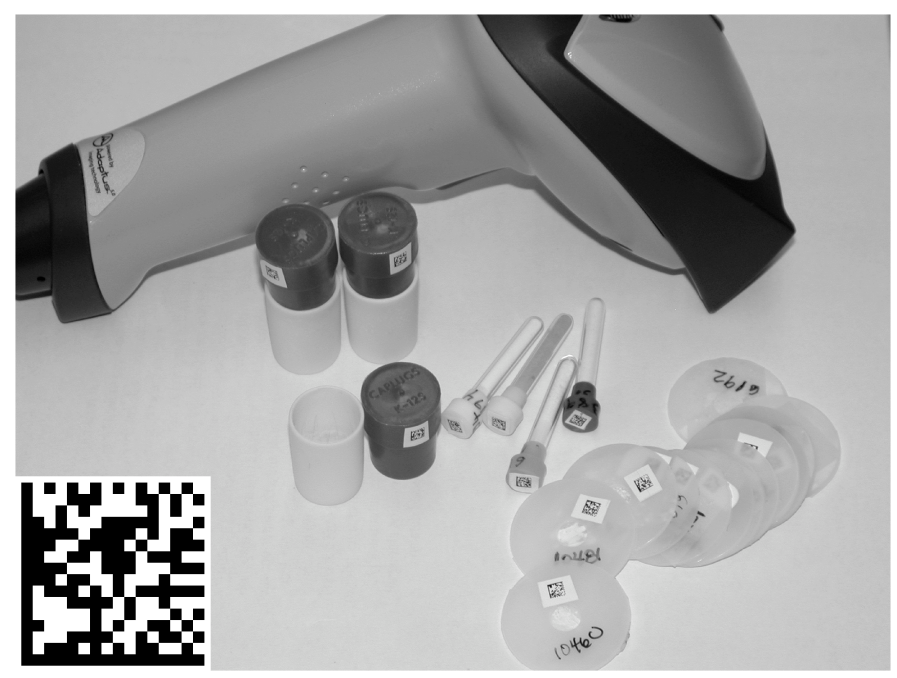

Fig. 4. Alumina crucibles for synthesis, fused silica cuvettes for measuring luminescence spectra and pulsed X-rays, and nylon washers for X-ray diffraction measurements. The hand-operated bar code reader (above) is used to read barcodes shown attached to each sample. Inset (lower left) shows enlarged 2D bar code.

\section{SAMPLE CHARACTERIZATION}

\section{A. X-ray Diffractometry}

A Nonius FR591 water-cooled rotating copper-anode X-ray generator $(50 \mathrm{kV}, 100 \mathrm{~mA})$ (Bruker AXS Inc., Madison, WI) is used to produce the diffraction pattern for each compound. Samples are sealed between two pieces of plastic adhesive tape, attached to a nylon washer, and loaded into a specially constructed 16-sample computer-controlled sample changer. Because this seal is not perfect, hygroscopic samples are measured promptly. The collimated $8 \mathrm{keV} \mathrm{X}$-rays that pass directly through the sample are absorbed by a lead beam stop. Diffracted X-rays are detected by a Mar detector plate (Mar USA, Inc., Evanston, IL), and the 2D image is transformed into a standard 1D diffraction pattern (Fig. 5) with "Fit2D" software [6]. "Match!" software [7] is then used to determine which compounds in the Powder Diffraction Database [1] best match the sample.

\section{B. X-ray Luminescence Spectroscopy}

The water-cooled X-ray generator described above has two exit beams. The first is used to produce diffraction patterns (described in the previous section), and the second beam is used to irradiate samples in fused silica cuvettes and record their spectral response.

To measure the luminescence of the $\mathrm{X}$-ray exposed samples, we use a SpectraPro-2150i spectrometer (Acton Research Corp., Acton, MA) coupled to a PIXIS:100B charge- coupled detector (CCD) (Princeton Instruments, Inc., Trenton, $\mathrm{NJ}$ ). The spectrometer has a motorized order-sorting filter wheel (with 4 filters), a motorized slit (which can be set from $0.010 \mathrm{~mm}$ to $3.0 \mathrm{~mm}$ ), and two diffraction gratings (blazed for $300 \mathrm{~nm}$ and $500 \mathrm{~nm}$, both with 300 lines $/ \mathrm{mm}$ ) mounted on a turret. The order-sorting filters eliminate all $2^{\text {nd }}$ or higher order peaks. The grating density allows the spectrometer to acquire a spectrum covering slightly over $500 \mathrm{~nm}$ with a single exposure. The CCD is thermoelectrically cooled to $-70^{\circ} \mathrm{C}$ (for low dark current) and has a $1340 \times 100$ pixel array, which corresponds to about $0.39 \mathrm{~nm}$ bandwidth for each pixel with the gratings described above.
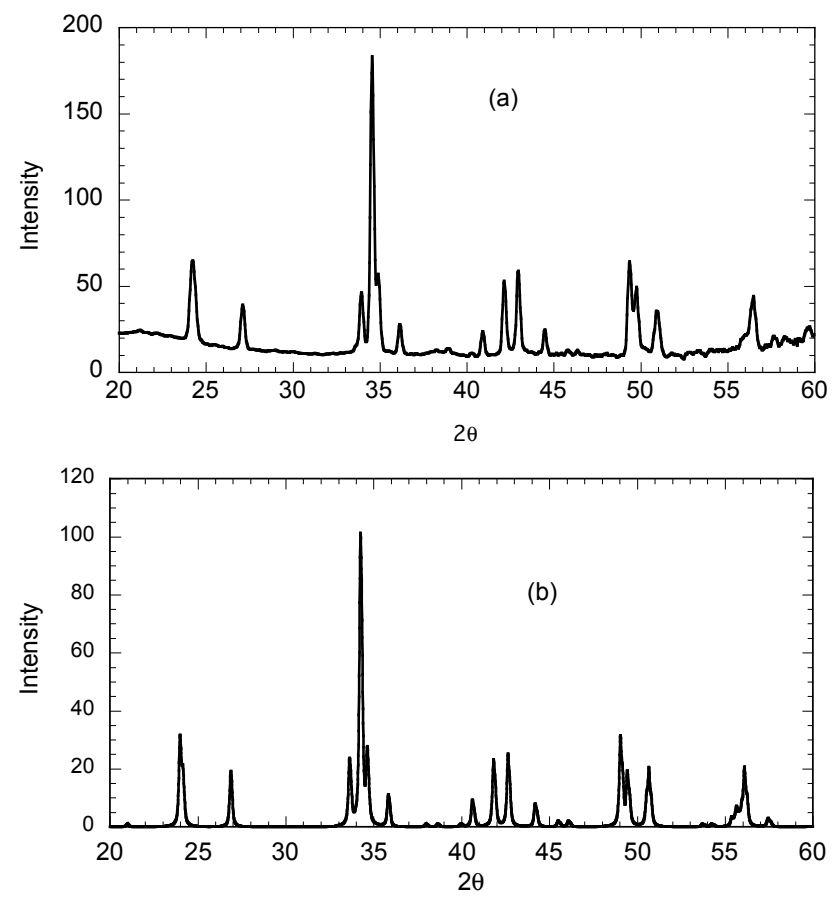

Fig. 5. Upper curve (a) is the diffraction pattern for $\mathrm{YAlO}_{3}$ measured in this work. Lower curve (b) is the diffraction pattern for $\mathrm{YAlO}_{3}$ from the Powder Diffraction Database [1].

The specially constructed sample changer has a bar code reader and places for 64 fused silica cuvettes. A LabVIEW program controls the sample changer and spectrometer, reads the barcodes, acquires data from the CCD, and transfers them to the on-line database.

For each crystal sample, a background spectrum is acquired with the CCD shutter closed, immediately followed by a blue spectrum $(200-360 \mathrm{~nm})$, a green spectrum $(360-620 \mathrm{~nm})$, and a red spectrum $(620-1000 \mathrm{~nm})$. All three spectra are acquired with the same slit width and the same exposure time. For the blue spectrum, we use the $300 \mathrm{~nm}$ blazed grating without any order-sorting filter and a center wavelength of $320 \mathrm{~nm}$. The green spectrum is acquired with the $500 \mathrm{~nm}$ blazed grating, a $320 \mathrm{~nm}$ cut-off, order-sorting filter and a center wavelength of $480 \mathrm{~nm}$. The red spectrum is acquired with the $500 \mathrm{~nm}$ blazed grating, a $590 \mathrm{~nm}$ cut-off order-sorting filter, and a center wavelength of $750 \mathrm{~nm}$. To calculate the resolving power of the spectrometer for each run, the slit width is multiplied with the 
linear dispersion, which is $19.7 \mathrm{~nm} / \mathrm{mm}$ (for the blue spectrum), $19.4 \mathrm{~nm} / \mathrm{mm}$ (for the green spectrum), and $18.9 \mathrm{~nm} / \mathrm{mm}$ (for the red spectrum). The three spectra are corrected for background, grating efficiency, and CCD quantum efficiency, and are stitched together to create one continuous spectrum covering $200 \mathrm{~nm}$ to $1000 \mathrm{~nm}$. The raw data, the correction factors, the final spectrum, as well as all parameters for the luminescence measurements are uploaded to the on-line database.

Fig. 6 shows the X-ray luminescence spectrum for $\mathrm{Y}_{2} \mathrm{O}_{2} \mathrm{~S}: \mathrm{Tb}$ using $200 \mu \mathrm{m}$ slits (3.9 $\mathrm{nm}$ spectral resolution). This sample is included in every set of samples loaded into the sample changer to monitor system performance.

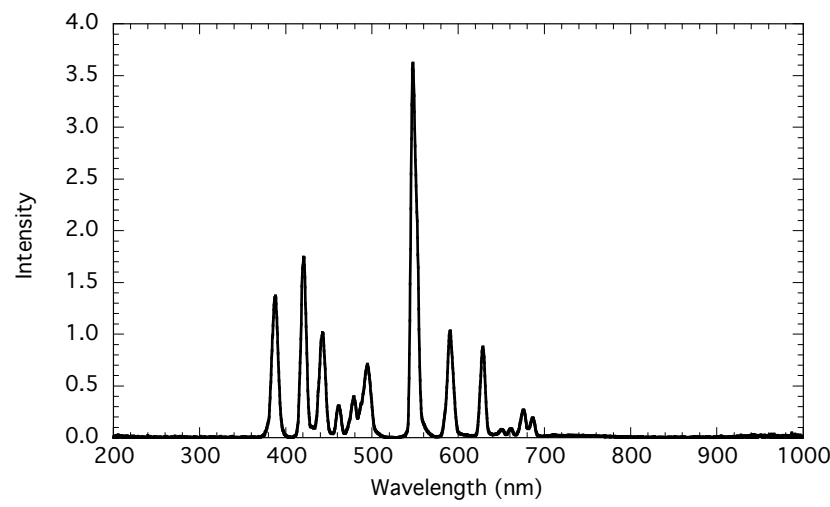

Fig. 6. X-ray luminescence spectrum of $\mathrm{Y}_{2} \mathrm{O}_{2} \mathrm{~S}$ : Tb measured in this work.

\section{Pulsed X-ray Time Response}

A pulsed X-ray system [8,9] is used to measure the time response and to determine the exponential decay components whose sum fits the measured data (Fig. 7). This system consists of a YAG:Nd laser that produces a $5 \mathrm{~W} 532 \mathrm{~nm}$ beam that drives a Ti-sapphire laser to produce 200 fs pulses at 800 nm (Newport Corporation, Spectra-Physics Lasers Division, Mountain View, CA). A portion of the $800 \mathrm{~nm}$ beam is sent to a fast diode (EOT, Inc., Traverse City, MI) with a 10 ps time jitter, and its output is used to start a Tennelec TC862 time-toamplitude converter (Canberra, Inc., Meriden, CT). The doubled $400 \mathrm{~nm}$ beam is sent to an N5084 light-excited X-ray tube (Hamamatsu, Inc., Hamamatsu, Japan). This X-ray tube is operated with a grounded cathode and an anode at $+30 \mathrm{kV}$. The average X-ray energy is $18 \mathrm{keV}$ [9]. An R3809U-50 microchannel phototube with 35 ps fwhm time jitter (Hamamatsu, Inc., Hamamatsu, Japan) and an Ortec 9307 pico-timing discriminator (Ortec, Inc., Oak Ridge, TN) are used to generate stop pulses from individual fluorescent photons. If the stop pulse rate is much less than the start pulse rate, then the spectrum of time-to-amplitude converter output pulses has the same shape as the time response of the scintillation light [10]. The impulse response of the system is 100 ps fwhm. Two excitation rates are used: $400 \mathrm{kHz}$ for measuring decay times below $5 \mu \mathrm{s}$, and $80 \mathrm{kHz}$ for measuring decay times below $25 \mu \mathrm{s}$. The typical average X-ray tube anode current is $0.6 \mu \mathrm{A}$ at $400 \mathrm{kHz}$ and $0.1 \mu \mathrm{A}$ at $80 \mathrm{kHz}$. Data are digitized into $8.1 \mathrm{ps}$ time bins at $400 \mathrm{kHz}$ and $40.5 \mathrm{ps}$ time bins at $80 \mathrm{kHz}$. At the conclusion of every measurement a sum of exponentials is automatically fitted to the data and the fitted parameters are uploaded into the on-line database. The decay time data are compressed by summing time bins in groups of 2, 4, 8, etc., for longer and longer times before and after the excitation pulse. Time bins within 250 bins of the excitation pulse are not compressed. In this way 64000 time bins are compressed into 2000 time bins for fitting.

The specially constructed sample changer has a bar code reader and places for 64 fused silica cuvettes. A LabVIEW program controls the sample changer, reads the barcodes, acquires data, transfers them to the on-line database, and initiates the component fitting process.

Fig. 8 shows the measured light response of $\mathrm{YAlO}_{3}: \mathrm{Ce}$. This sample is included in every set of samples loaded into the sample changers to monitor system performance. Fitted exponential decay times are $27 \mathrm{~ns}(70 \%), 95 \mathrm{~ns}(15 \%)$, and $1700 \mathrm{~ns}(15 \%)$. For a 1 million-event dataset the value of chi squared is typically about 2400 , or 1.2 per degree of freedom. The microchannel PMT signal rate normalized by the X-ray current is used to estimate luminosity. The apparent luminosity of a crystalline powder sample depends not only on the intrinsic luminosity of the sample but also on other factors such as the particle size, the X-ray attenuation length, and the index of refraction. Table I compares the accepted luminosity in photons/MeV with the photomultiplier tube counts per second divided by the $\mathrm{X}$-ray tube anode current in $\mu \mathrm{A}$ for a number of known scintillators. As expected, the ratio is not constant but varies by a factor of two from the average. This accuracy is adequate for screening materials to discover bright new scintillators.

Any material with an observed luminosity above a few thousand counts/s/ $\mu \mathrm{A}$ is synthesized under various conditions and with various dopant concentrations to maximize the luminosity.

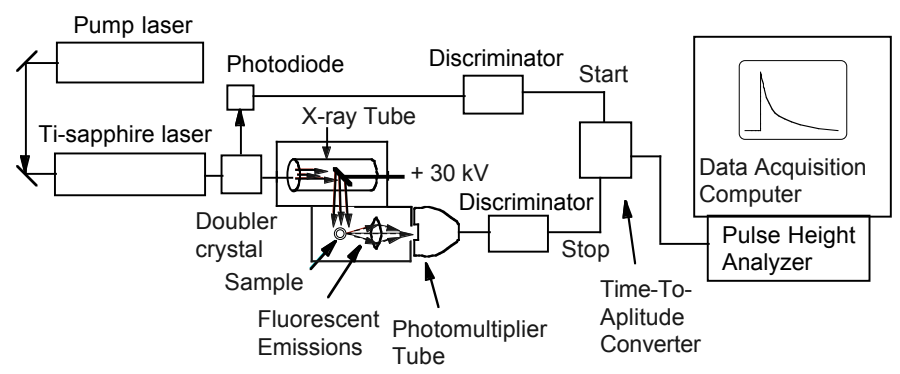

Fig. 7. Pulsed X-ray facility. A light-excited X-ray tube converts pulses from an ultra-fast laser into $80 \mathrm{ps}$ pulses of X-rays. A time-to-amplitude converter transforms the arrival times of fluorescent photons into the time response of the scintillation emissions of the sample. 


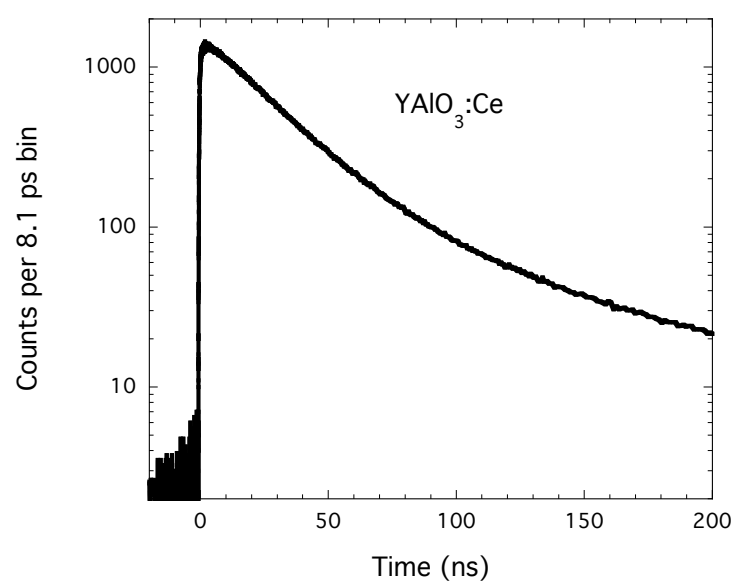

Fig. 8. Pulsed X-ray time response of the scintillation light from $\mathrm{YAlO}_{3}: \mathrm{Ce}$

TABLE I

CRYSTAL VS. POWDER LUMINOSITIES

\begin{tabular}{lccc}
\hline Scintillator & $\begin{array}{c}\text { Luminosity } \\
\text { (photons/MeV) }\end{array}$ & $\begin{array}{c}\text { Pulsed X-ray } \\
\text { (counts/s/ } \mu \mathrm{A} \text { )* }\end{array}$ & Ratio \\
\hline $\mathrm{BaF}_{2}$ & 10,400 & 7,200 & 1.45 \\
$\mathrm{Bi}_{4} \mathrm{Ge}_{3} \mathrm{O}_{12}$ & 8,200 & 7,000 & 1.15 \\
$\mathrm{CaWO}_{4}$ & 6,000 & 3,100 & 1.9 \\
$\mathrm{CeF}_{3}$ & 4,500 & 1,000 & 4.5 \\
$\mathrm{CsF}$ & 1,900 & 1,100 & 1.7 \\
$\mathrm{PbWO}_{4}$ & 100 & 46 & 2.2 \\
$\mathrm{YAlO}_{3}: \mathrm{Ce}$ & 21,000 & 10,000 & 2.1 \\
$\mathrm{ZnWO}_{4}$ & 21,500 & 6,200 & 3.5 \\
\hline
\end{tabular}

*Typical X-ray tube current $0.6 \mu \mathrm{A}$

\section{Database Management System}

All synthesis and measurement data are stored and organized in an on-line database management system, FileMaker Server Advanced (FileMaker, Inc., Santa Clara, CA). Researchers use FileMaker Pro client software for viewing and retrieving the data. Custom software created with LabVIEW, PHP, and Perl use the ODBC (Open Database Connectivity) interface for automated data upload as well as retrieval for automated analysis processes. Promising samples can be readily identified by searching on luminosity and decay times.

The information available for each sample includes:

1) Weights, sources, and purities of component chemicals

2) Synthesis procedures, such as furnace identification, atmosphere, temperature vs. time profile, and differential thermal analysis, if performed.

3) Sample number and barcodes

4) X-ray diffraction (XRD) pattern

5) Crystalline phases determined from the match of the sample XRD to the Powder Diffraction Database

6) X-ray luminescence spectrum, component wavelengths, and intensities

7) Pulsed X-ray time response data, exponential decay times, and fractions

\section{DISCUSSION}

The facility described was designed to synthesize and characterize large numbers of crystalline powder samples with much less effort than growing crystals. During the past year over 900 samples have been processed, representing 150 different compounds. Results will be presented at a later date.

Barcodes are used to keep track of the samples through all the synthesis and characterization processes. Measurements of emission wavelengths, decay times, and fractions are reliable, while the luminosity is less certain due to scattering and loss of light among the small crystals of the powder. The effort of growing crystals is reserved for the few successful samples found by powder measurements. Such crystals then enable accurate measurements of luminosity, proportionality, and energy resolution.

\section{ACKNOWLEDGMENT}

We thank R. Nordmeyer and E. Cornell for designing and building the three automated sample changers and for providing control software.

This document was prepared as an account of work sponsored by the United States Government. While this document is believed to contain correct information, neither the United States Government nor any agency thereof, nor The Regents of the University of California, nor any of their employees, makes any warranty, express or implied, or assumes any legal responsibility for the accuracy, completeness, or usefulness of any information, apparatus, product, or process disclosed, or represents that its use would not infringe privately owned rights. Reference herein to any specific commercial product, process, or service by its trade name, trademark, manufacturer, or otherwise, does not necessarily constitute or imply its endorsement, recommendation, or favoring by the United States Government or any agency thereof, or The Regents of the University of California. The views and opinions of authors expressed herein do not necessarily state or reflect those of the United States Government or any agency thereof or The Regents of the University of California.

\section{REFERENCES}

[1] PDF, Powder Diffraction File, International Centre for Diffraction Data, Newtown Square, PA, U.S.A., 2006.

[2] ICSD, Inorganic Crystal Structure Database, Fachinformationszentrum Karlsruhe, Karlsruhe, Germany, 2006.

[3] N. Cherepy, personal communication, January, 2007.

[4] M. Payne, M. P. Teter, D. C. Allan, et al., "Iterative minimization techniques for ab initio total-energy calculations: molecular dynamics ad conjugate gradients," Rev Mod Phys, vol. 64, pp. 1045-1097, 1992.

[5] A. Canning, A. Chaudhry, L.-W. Wang, et al., "Electronic structure studies and predictions for new Ce-doped gamma detector materials," IEEE Nuclear Science Symposium Conference Record, pp. 2466-2468, 2007.

[6] A. P. Hammersley, FIT2D V12.012 Reference Manual V6.0. ESRF International Report No. ESRF98HA01T. Program available at http://www.esrf.eu/computing/scientific/FIT2D/, 2004.

[7] Match!, Crystal Impact, K. Brandenburg \& H. Putz GbR, Bonn, Germany, 2007.

[8] S. E. Derenzo, W. W. Moses, S. C. Blankespoor, et al., "Design of a pulsed x-ray system for fluorescent lifetime measurements with a timing resolution of 109 ps," IEEE Trans Nucl Sci, vol. NS-41, pp. 629-631, 1994.

[9] S. C. Blankespoor, S. E. Derenzo, W. W. Moses, et al., "Characterization of a pulsed X-ray source for fluorescent lifetime measurements," IEEE Trans Nucl Sci, vol. 41, pp. 698-702, 1994. 
[10] L. M. Bollinger and G. E. Thomas, "Measurement of the time dependence of scintillation intensity by a delayed-coincidence method," Rev Sci Instr, vol. 32, pp. 1044-1050, 1961. 